\title{
CARACTERIZACIÓN DE LAS COMPETENCIAS TRANSVERSALES DE LOS DOCENTES DEL BACHILLERATO UNIFICADO EN EL CENTRO EDUCATIVO MIRAFLORES DE LA CIUDAD DE GUAYAQUIL
}

\author{
CHARACTERIZATION OF CROSS SKILLS OF TEACHERS OF HIGH \\ SCHOOL EDUCATION CENTER UNIFIED IN MIRAFLORES CITY \\ GUAYAQUIL
}

Dra. Susana Marlene Hinojosa Dazza, MSc.

shinojosad@ulvr.edu.ec

Universidad Laica VICENTE ROCAFUERTE de Guayaquil

Ing. Rosa Dolores Hinojosa Dazza, MSc.

Universidad Laica VICENTE ROCAFUERTE de Guayaquil

\section{RESUMEN}

El desarrollo de competencias transversales de los docentes es hoy un tema de máximo interés; estas permiten la movilización integrada de conocimientos, habilidades, hábitos, componentes metacognitivos, rasgos de la personalidad y valores todos en función de ejecutar acciones positivas en situaciones cambiantes del proceso pedagógico. El desarrollo de competencias transversales de los docentes del bachillerato unificado en el Centro Educativo Miraflores (CEM) de la ciudad de Guayaquil, es el centro de este artículo. Su objetivo es exponer los resultados que se han obtenido en la caracterización del nivel de desarrollo de dichas competencias en la institución educativa mencionada. Para ello, se emplearon métodos nivel empírico como la observación a docentes, encuesta a docentes y a estudiantes; $y$, entrevista a autoridades e informantes clave. Los resultados confirmaron insuficiencias en las competencias relacionadas con el enfrentamiento y respuestas a las situaciones educativas que se le presentan a los docentes, la organización de estrategias de solución, que faciliten actuar frente a dichas situaciones educativas, en la aplicación de estrategias de solución a las problemáticas que enfrentan los docentes. así como en la disposición a la colaboración, la ejecución exitosa de tareas de en conjunto y el estímulo al trabajo en equipo, la identificación de los problemas educativos, la identificación de las alternativas de solución viables que permitan dar solución a los problemas educativos, la implementación de alternativas de solución y la evaluación de alternativas de solución.

Palabras clave: competencias, competencia profesional, competencias transversales, formación de docentes

\begin{abstract}
The development of skills of teachers is today a topic of interest. Enable the integrated knowledge, skills, and habits, mobilization of knowledge, metacognitive components, personality traits and values all according to take positive action on changing situations of the learning process. The development of skills of teachers in the unified baccalaureate in the Miraflores Education Centre (CEM) of the city of Guayaquil is the center of attention in this article. Its goal is to expose the results that have been obtained in the characterization of the level of development these skills in the aforementioned educational institution. For this reason, empirical methods such as: teachers'observations, survey to teachers and students and interview with authorities and key informants were used. The results confirmed inadequacies in skills related to the confrontation and responses to an educational situation that they are presented to the teachers, the organization of solution strategies that facilitate acting with these educational situations, the implementation of strategies of solution to the problems faced by teachers as well as disposition to collaboration, the successful execution of tasks of altogether and the encouragement of teamwork, the identification of educational problems the identification of viable alternatives of solution that will allow to solve the educational problems, the implementation of alternative solutions and the evaluation of alternative solutions.
\end{abstract}

Key words: Competencies, professional competencies, transversal competencies, profesional development. 


\section{Introducción}

El mundo de hoy demanda del ser humano, su preparación en todos los órdenes. Las instituciones requieren de su capital humano ser eficientes y eficaces en su trabajo para dar respuesta a las demandas más exigentes del entorno económico, político y social. A esta realidad no escapa el sector educacional. Es necesario brindar una educación de calidad.

Ecuador, país que avanza también en la búsqueda de la calidad educativa ha dejado plasmada sus intenciones en la Constitución de la República (2008), donde en su artículo 27, expresa que: "La educación se centrará en el ser humano y garantizará su desarrollo holístico, (...) estimulará el (...) desarrollo de competencias y capacidades para crear y trabajar."

A su vez, la Ley Orgánica de Educación Intercultural (2011), en su artículo 22, literal dd, señala, que se tendrán en cuenta estándares e indicadores de calidad educativa, los cuales estarán referidos al rendimiento académico estudiantil y al desempeño de las y los docentes.

Teniendo en cuenta estos presupuestos, el Ministerio de Educación de Ecuador presentó en el año 2012 los Estándares de Calidad Educativa. Los estándares de calidad referidos al desempeño profesional de los docentes son de particular importancia, en tanto los docentes, junto a los estudiantes son los principales actores del proceso pedagógico.

En el Bachillerato General Unificado, los estándares de calidad de desempeño profesional docente se enlazan directamente con los objetivos del nivel: preparar a los estudiantes para la vida y la participación en una sociedad democrática, la continuidad de estudios y para el mundo laboral y el emprendimiento, por lo que es necesario desarrollar en estos docentes competencias profesionales que les permitan obtener resultados satisfactorios en su labor, entre las que se encuentran las competencias transversales.

El desarrollo de competencias transversales de los docentes del Bachillerato General Unificado en el Centro Educativo Miraflores (CEM) de la ciudad de Guayaquil, ha llamado la atención de estas autoras, las que a partir de la observación sistemática por más de 25 años como docentes de este nivel y del nivel superior, han identificado una serie de insuficiencias que indican un limitado desarrollo de las competencias transversales en estos docentes. Estas insuficiencias se centran en:
- La limitada posibilidad de resolver problemas, es decir, enfrentarse y dar respuesta a una situación determinada.

- La falta de disposición para trabajar en equipo, para lograr la interdisciplinariedad y para colaborar de manera coordinada en las tareas.

- Limitaciones en la implementación de estrategias de enseñanza aprendizaje que promuevan la participación y el debate.

- Escasas expectativas respecto al aprendizaje de sus estudiantes, es decir, que esto no solo se refiera a los conocimientos y habilidades sino que considere como fundamental, promover valores y el ejercicio de los derechos humanos en el marco del Buen Vivir.

El estudio profundo del material bibliográfico que existe acerca de las competencias, las competencias profesionales (Aguiar, 2005; González y Álvarez, 2012; Jofré, 2009) y dentro de ellas las transversales (Barriga, 2005; Solé-Pareta, Domingo-Pascual y Santos-Boada, 2014; Torrecilla, Martínez, Olmos y Rodríguez, 2014), realizado como parte de la investigación permitió a las autoras considerar que las competencias transversales en los docentes, son aquellas competencias que "movilizan integradamente conocimientos, habilidades, hábitos, componentes metacognitivos, rasgos de la personalidad y valores adquiridos en función de ejecutar acciones positivas en situaciones cambiantes del proceso pedagógico y dentro de este, en el proceso de enseñanza-aprendizaje." (Hinojosa, 2015).

Dentro de las competencias transversales más necesarias a ser logradas en los docentes del Bachillerato General Unificado, de acuerdo a las exigencias educativas al nivel, al trabajo profesional de los docentes y a la calidad que se espera en el aprendizaje de los estudiantes, se encuentran las siguientes:

- Competencia para la resolución de problemas, que se centra en los conocimientos y habilidades para enfrentarse y dar respuesta a una situación determinada, unido a la posibilidad de organizar y aplicar estrategias o secuencias de acciones para encontrar la solución.

- Competencia para implicarse responsablemente en el trabajo, se trata de la responsabilidad desarrollada como valor en la persona con la subsiguiente actitud que lo exprese en las situaciones laborales, profesionales y personales. 
- Competencia para trabajar en equipo, que incluye la disposición para colaborar de manera coordinada en la tarea realizada con un equipo de personas para conquistar un objetivo propuesto.

- Competencia relacionada con la autonomía, es decir, poder realizar una tarea de forma independiente sin necesidad de recibir ayuda o con la menor cantidad de ayudas posibles.

- La competencia para relacionarse con los otros, que es la disposición para comunicarse con los otros con el trato adecuado, con atención y simpatía.

- Competencia dirigida a la iniciativa y disposición para tomar decisiones sobre propuestas o acciones, que en dependencia de su grado de complejidad puede llegar a ser innovación.

De acuerdo con el análisis que antecede se em- prendió la investigación cuyo problema científico se identificó del siguiente modo: ¿cómo influye el desarrollo de competencias transversales en los docentes del Bachillerato General Unificado del CEM de la ciudad de Guayaquil en el mejoramiento del aprendizaje de los estudiantes?

\section{Objetivo}

El objetivo del presente artículo es exponer los resultados que se han obtenido en la caracterización del nivel de desarrollo de las competencias transversales de los docentes del CEM de la ciudad de Guayaquil.

\section{Materiales y Métodos}

Para realizar la caracterización del desarrollo de las competencias transversales de los docentes de CEM, se operacionalizó esta variable en seis dimensiones con sus correspondientes indicadores, como se refleja en la Tabla 1.

\section{Tabla 1. Operacionalización de la variable desarrollo de competencias transversales en los docentes.}

\begin{tabular}{|c|c|}
\hline Dimensiones & 100 \\
\hline \multirow{3}{*}{$\begin{array}{l}\text { 1. Resolución de problemas: Ha- } \\
\text { bilidades para enfrentarse y dar } \\
\text { respuesta las situaciones que se le } \\
\text { presentan y organizar y aplicar es- } \\
\text { trategias para ello. }\end{array}$} & $\begin{array}{l}\text { 1.1. Enfrentamiento y respuesta a las situaciones educativas: Habilidad para enfrentar- } \\
\text { se y dar respuesta a una situación educativa determinada }\end{array}$ \\
\hline & $\begin{array}{l}\text { 1.2. Organización de estrategias de solución: Posibilidad de organizar estrategias para } \\
\text { encontrar la solución a los problemas }\end{array}$ \\
\hline & $\begin{array}{l}\text { 1.3. Aplicación de estrategias de solución: Posibilidad de aplicar estrategias para en- } \\
\text { contrar la solución a los problemas }\end{array}$ \\
\hline \multirow{3}{*}{$\begin{array}{l}\text { 2. Implicación responsable en el } \\
\text { trabajo: Responsabilidad y actitud } \\
\text { personal ante el trabajo y la vida } \\
\text { profesional. }\end{array}$} & $\begin{array}{l}\text { 2.1. Responsabilidad: Actuación consciente del docente ante las obligaciones o debe- } \\
\text { res inherentes a su vida laboral y profesional. }\end{array}$ \\
\hline & $\begin{array}{l}\text { 2.2. Actitud personal ante las situaciones laborales: evaluación positiva o negativa que } \\
\text { el docente hace sobre su trabajo que se expresa en la satisfacción, la participación en } \\
\text { el trabajo y el compromiso con la institución. }\end{array}$ \\
\hline & $\begin{array}{l}\text { 2.3. Actitud personal ante las situaciones profesionales: evaluación positiva o negativa } \\
\text { que el docente hace sobre su profesión que se expresa en la satisfacción hacia la pro- } \\
\text { fesión, el orgullo con ella y el compromiso por mantenerse vinculado a ella. }\end{array}$ \\
\hline \multirow{3}{*}{$\begin{array}{l}\text { 3. Trabajo en equipo: Se involucra } \\
\text { en tareas colectivas de manera } \\
\text { efectiva y estimula a sus colegas y } \\
\text { estudiantes a trabajar en equipo. }\end{array}$} & 3.1 Disposición a la colaboración: Forma en que se involucra en las tareas colectivas. \\
\hline & $\begin{array}{l}\text { 3.2. Ejecución exitosa de tareas en conjunto: Colaboración efectiva con equipos de } \\
\text { personas para conquistar un objetivo propuesto. }\end{array}$ \\
\hline & $\begin{array}{l}\text { 3.3 Estímulo al trabajo en equipo: Formas que emplea para estimular en sus colegas y } \\
\text { estudiantes el trabajo en equipo. }\end{array}$ \\
\hline \multirow{2}{*}{$\begin{array}{l}\text { 4.Autonomía: realiza tareas con po- } \\
\text { cas ayudas y estimula el trabajo in- } \\
\text { dependiente en sus estudiantes. }\end{array}$} & $\begin{array}{l}\text { 4.1.Independencia: Realización de las tareas con la menor cantidad de ayudas posi- } \\
\text { bles. }\end{array}$ \\
\hline & $\begin{array}{l}\text { 4.2. Estimulación al trabajo autónomo: Forma en que estimula la realización de tareas } \\
\text { independientes en sus estudiantes. }\end{array}$ \\
\hline \multirow{2}{*}{$\begin{array}{l}\text { 5.Iniciativa: predisposición a enfre- } \\
\text { nar las tareas y situaciones y actitud } \\
\text { proactiva en la realización de estas }\end{array}$} & $\begin{array}{l}\text { 5.1.Predisposición: propensión a emprender acciones, crear oportunidades y mejorar } \\
\text { resultados sin estímulo externo. }\end{array}$ \\
\hline & $\begin{array}{l}\text { 5.2. Proactividad: adopción de una actitud positiva ante la realidad y dar el paso adelan- } \\
\text { te para resolver las problemáticas que se presentan }\end{array}$ \\
\hline \multirow{4}{*}{$\begin{array}{l}\text { 6. Toma de decisiones: identifica las } \\
\text { situaciones conflictivas, las alternati- } \\
\text { vas de solución, las selecciona, im- } \\
\text { plementa y evalúa. }\end{array}$} & $\begin{array}{l}\text { 6.1. Identificación de los problemas educativos: encuentra las situaciones educativas } \\
\text { conflictivas tanto en el aula de clases como en el ámbito laboral en general. }\end{array}$ \\
\hline & $\begin{array}{l}6.2 \text { Identificación de las alternativas de solución: encuentra alternativas del aula de } \\
\text { clases como en ámbito educativo en general. }\end{array}$ \\
\hline & 6.3. Implementación de las alternativas: aplica la alternativa seleccionada. \\
\hline & 6.4. Evaluación de la alternativa: va \\
\hline
\end{tabular}


A partir de estos indicadores, se elaboraron los instrumentos correspondientes para obtener la información. Estos fueron: guía de observación a docentes, cuestionario de encuesta a docentes y a estudiantes; y, guía de entrevista a autoridades e informantes clave.

El Centro Educativo Miraflores cuenta con 182 estudiantes cursando el bachillerato unificado, 15 docentes que imparten clases en este nivel, 4 autoridades y se consideraron 5 informantes clave, de ellos, tres asesores para el trabajo docente-metodológico y dos directores de ciclo. (Se consideró como población en las autoridades, los directores de los ciclos de media y bachillerato unificado).
El estudio se realizó con el 100\% de las autoridades e informantes claves, por lo que no se seleccionó población. Para la selección de la muestra de estudiantes y docentes, se empleó la ecuación siguiente:

$$
n=\frac{N \sigma^{2} Z^{2}}{(N-1) e^{2}+\sigma^{2} Z^{2}}
$$

Los cálculos realizados permitieron determinar el tamaño de la muestra de estudiantes y docentes. La Tabla 2 muestra los datos referidos a población y muestra empleados en el diagnóstico inicial.

Tabla 2. Datos de población y muestra.

\begin{tabular}{|l|c|c|c|c|}
\hline \multicolumn{1}{|c}{ Grupos humanos investigados } & Población & 8 & & Aleatorio simple \\
\hline Docentes de bachillerato unificado & 15 & 42 & & Aleatorio estratificado \\
\hline Estudiantes del bachillerato & 182 & - & 100 & \\
\hline Autoridades & 4 & - & 100 & \\
\hline Informantes clave & 5 & & & \\
\hline
\end{tabular}

Se aplicaron los instrumentos diseñados, se procesó la información proveniente de estos y se realizó el análisis porcentual y cálculo de frecuencias de aparición de los valores de acuerdo con los criterios utilizados. La triangulación metodológica permitió contrastar los resultados de los instrumentos y arribar a conclusiones.

\section{Resultados}

La dimensión Resolución de problemas se encuentra en el estado no deseado, con la mayoría de las frecuencias $(42,7)$ calculadas en nivel bajo y 37,8 en nivel medio (alejado de lo deseado). En el nivel alto (estado deseado), solo hay 19,5 frecuencias de aparición de este nivel.

Estos resultados son coincidentes, tanto en la observación a docentes como en las opiniones de los estudiantes, los que mayoritariamente, consideran que la Resolución de problemas presenta insuficiencias en cómo los docentes enfrentan y dan respuesta a las problemáticas educativas, así como en la organización de estrategias para la solución de problemas y la aplicación de estas.

\section{Estos resultados pueden observarse en la Figura 1.}

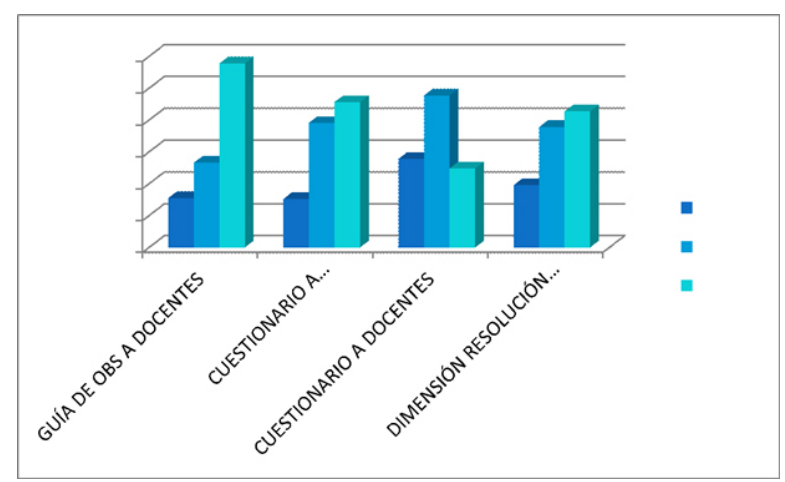

Figura 1. Dimensión Resolución de problemas. Resultados de triangulación metodológica.

Sin embargo, la dimensión Implicación responsable en el trabajo se comporta en el estado deseado tanto en la observación a docentes como las opiniones de estudiantes y de los docentes. No obstante estos resultados altos, debe notarse que se mantienen respuestas que evalúan esta dimensión en niveles, en general, alejados de lo deseado. Las frecuencias de aparición de estos niveles medio y bajo, es alto $(62,1)$ en la guía de observación, en las opiniones de los estudiantes es de 55 , y en las de los docentes es de 50. 
Los resultados de la dimensión Implicación responsable en el trabajo constituyen una fortaleza para el desarrollo de las competencias transversales, puesto que los docentes evidencian responsabilidad y una actitud personal positiva ante las situaciones laborales y profesionales. Estos resultados pueden observarse en la Figura 2.

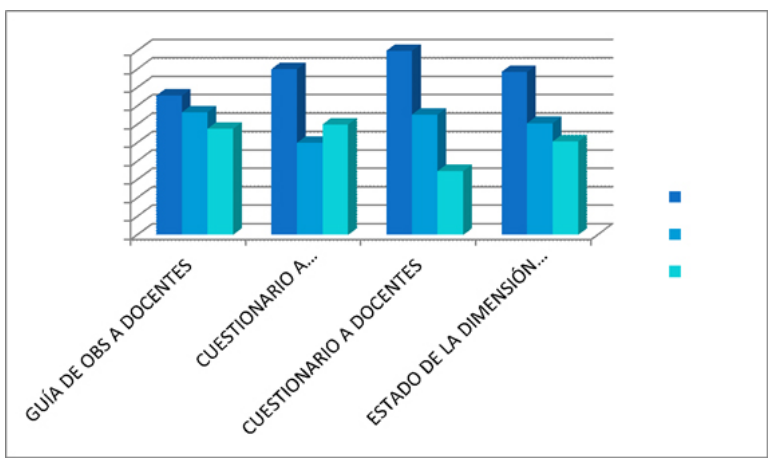

Figura 2. Dimensión Implicación responsable en el trabajo. Resultados de la triangulación metodológica.

El Trabajo en Equipo también se encuentra, en general, en el estado deseado aunque los resultados no coinciden en las observaciones realizadas y en las opiniones de docentes y estudiantes. Las observaciones reflejan bajos resultados, (55,5 de las frecuencias en el nivel bajo), no así las opiniones de estudiantes y docentes que coinciden en considerar en nivel alto $(39,1$ y 50 , respectivamente).

No obstante este resultado, deseado para la dimensión a partir de la triangulación, cuando se suman las frecuencias para niveles medio y bajo en cada instrumento, se encuentra que en las opiniones de los estudiantes hay 60,8 frecuencias y en las de los docentes, 60 . Ello indica que aún hay valores no deseados en la disposición a la colaboración y la ejecución exitosa de tareas en conjunto, competencias muy importantes para lograr calidad del aprendizaje en los estudiantes. Estos resultados pueden observarse en la Figura 3 .

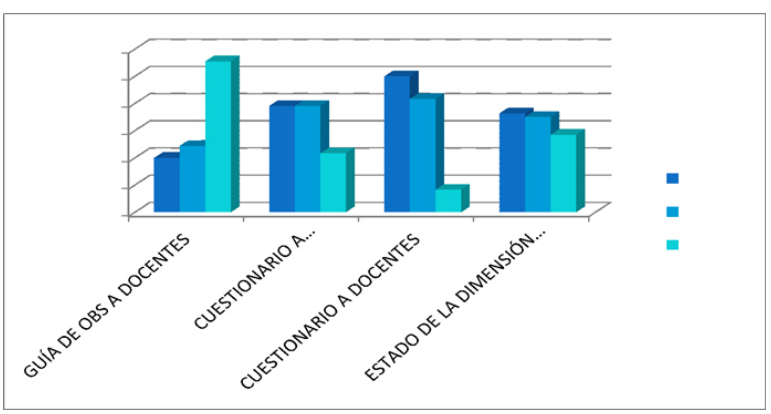

Figura 3. Dimensión Trabajo en equipos. Resultados de la triangulación metodológica.
La dimensión Autonomía, del mismo modo que la anterior se encuentra a partir de la triangulación metodológica en el estado deseado, en esto concuerdan las observaciones realizadas a los docentes y las opiniones de los estudiantes, con 50 y 58,7 de aparición de las frecuencias para este nivel deseado. Sin embargo, en opinión de los docentes, se aleja de lo deseado, con 46,9 de las frecuencias en nivel medio y 53,1 en el nivel bajo. Ninguna frecuencia en nivel alto.

La triangulación metodológica evidencia en general, aunque la dimensión se encuentra con 36,2 de las frecuencias calculadas en el estado deseado, hay un 63,8 de las frecuencias, la mayoría que tienden a lo no deseado, por lo tanto, la independencia y el estímulo para el trabajo en equipos, aún debe ser atendida prioritariamente para que influya en el desarrollo de las competencias transversales de los docentes y en la calidad del aprendizaje de los estudiantes. Estos resultados pueden observarse en la Figura 4.

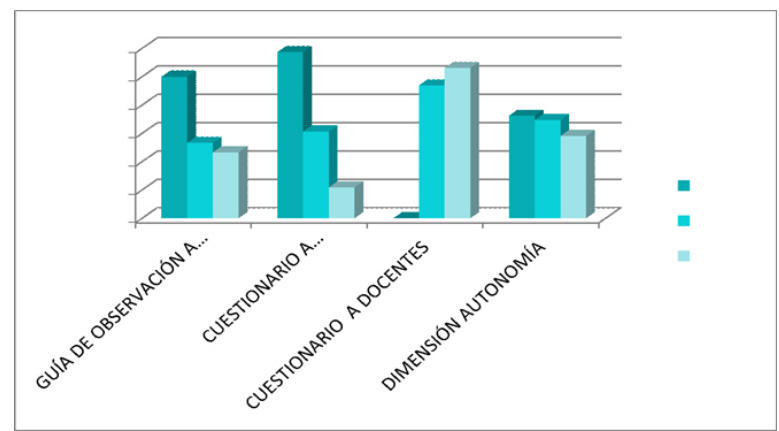

Figura 4. Dimensión Autonomía. Resultados de la triangulación metodológica

La dimensión Iniciativa se encuentra en el estado no deseado. Tanto en las observaciones a los docentes como en las opiniones de estos, coinciden los resultados que tienden a lo no deseado. (Solo 19,6 frecuencias en el nivel alto.)

Están afectadas, tanto la predisposición como la proactividad. Los resultados de la dimensión Iniciativa, en opinión de esta autora, se reflejan en los resultados que exhibe la dimensión Resolución de problemas, puesto que la predisposición y proactividad están relacionadas con las posibilidades que posee un docente para enfrentarse a los problemas, organizar estrategias para su solución u aplicarlas. Los resultados de la dimensión Iniciativa pueden observarse en la Figura 5. 


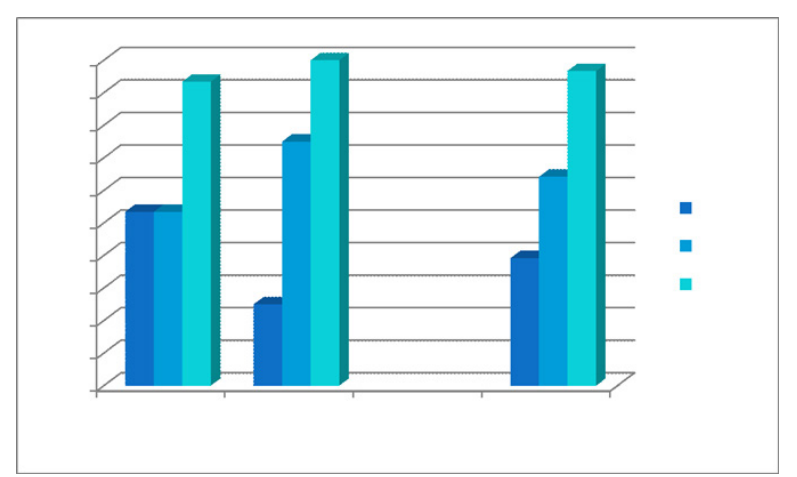

Figura 5. Dimensión Iniciativa. Resultados de la triangulación metodológica.

El análisis anterior también es coincidente con los resultados de la dimensión Toma de decisiones la cual se encuentra también en el estado no deseado, información que proviene de la observación a docentes y de sus opiniones (55 y 41,1 respectivamente en nivel bajo). En este sentido, se encuentran afectaciones en la identificación de los problemas educativos, la identificación de alternativas de solución, la implementación de dichas alternativas y su evaluación, todos estos aspectos importantes y relacionados con la iniciativa y la resolución de problemas. Estos resultados pueden observarse en la Figura 6.

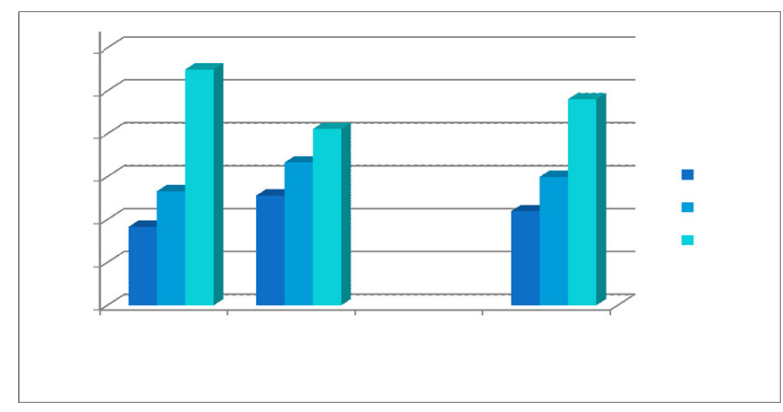

Figura 6. Dimensión Toma de decisiones. Resultados de la triangulación metodológica.

\section{Entrevista a autoridades y a informantes clave.}

La entrevista a autoridades y a informantes clave se realizó el 16 de enero del 2015, con el objetivo de profundizar en los resultados obtenidos en los anteriores instrumentos. Participaron las cinco autoridades de la institución y cuatro informantes claves (asesores metodológicos y jefes de ciclos). De ellos, uno tiene experiencia menor de 5 años, seis se encuentran en el rango de 5 a 10 años, y dos tienen más de 10 años de experiencia como directivos o asesores.

La primera parte de la entrevista se dirigió a las opiniones respecto al desarrollo de las competencias transversales en los docentes de bachillerato unificado. En este sentido las opiniones fueron:

- Algunos docentes presentan dificultades para enfrentarse y dar respuesta a una situación educativa determinada, por lo que les cuesta solucionar los problemas que se les presentan, no siempre son hábiles en organizar estrategias para encontrar la solución a los problemas y para aplicarlas. En general, las habilidades en este sentido no están en el valor que se desea. Les cuesta dominar los grupos y no tienen ese compromiso institucional.

- La responsabilidad ante las tareas laborales y profesionales en ocasiones también se afecta, puesto que muchos docentes no se sienten comprometidos con la institución, dan sus clases (Matemática, Lengua y Literatura) y con eso les basta. Es decir, en algunos docentes, la actitud que asumen ante las situaciones laborales que se les puedan presentar (conflictivas, o donde tengan que tomar decisiones importantes), no siempre es positiva, tienen dificultades para enfrentarlas, así como las que se les presentan en relación con situaciones profesionales, es decir, la actitud ante las acciones que realiza la institución en relación con la capacitación, no es que no asistan, es que asisten solo por tener la asistencia. Ante la primera dificultad que se les presenta, se retiran, no han desarrollado esa habilidad o competencia relacionada con sobreponerse ante las dificultades laborales o profesionales que se les puedan presentar en un momento determinado.

- Los docentes se involucran en las tareas colectivas, colaboran en gran medida en equipos de personas para conquistar un objetivo propuesto y estimulan en sus estudiantes el trabajo en equipo, sin embargo, no siempre estimulan en sus colegas de esta necesidad de trabajo en equipo, las acciones que los docentes realizan muchas veces son aisladas. Opinan que esto tiene mucho que ver con la selección del personal que va a impartir docencia en la institución y con la preparación pedagógica que poseen, muchas veces esa es nula. No siempre se logra que desarrollen estas acciones dirigidas no solo a la parte cognitiva, sino también a la parte procedimental y a la parte actitudinal, existen muchas falencias en el desarrollo de competencias para estimular en los estudiantes, sus competencias para el Siglo XXI.

- Se presentan dificultades en el estímulo en los jóvenes de la parte motivacional y afectiva, se concentran en su materia y los otros aspectos no se desarrollan o estimulan. Encuentran muchas insuficiencias en el compromiso de los 
docentes con el estímulo a la calidad del aprendizaje de sus estudiantes, solo se concentran en dar su clase y no hay esa visión hacia qué hacen los jóvenes en la clase, cómo se expresan, cómo se desempeñan. No siempre se estimula el aprendizaje crítico y valorativo en los estudiantes ni se acompaña a los estudiantes en su aprendizaje.

- La iniciativa propia en los docentes tiene falencias, no todos los docentes presentan posibilidades reales para emprender acciones, crear oportunidades y mejorar resultados sin estímulo externo.

- Señalan que hay mucho personal nuevo, y el compromiso con el trabajo en ese personal nuevo debe llegar con el tiempo, de sentirse parte de la institución. Es necesario reforzar en las capacitaciones, no solo lo relacionado con los contenidos de las materias, sino temas que ayuden a integrar a los nuevos y a los antiguos, que vayan más al desarrollo del compromiso, la responsabilidad ante sus deberes y ante la institución.

- En las observaciones que se ha realizado, se visualiza que los docentes no estimulan en sus alumnos esa conexión con la realidad, tan necesaria en la época, y ello se debe, en buena medida a que no se encuentran preparados para la labor docente. No siempre adoptan una actitud positiva ante la realidad y dan el paso adelante para resolver las problemáticas que se presentan. Muchas veces, evaden las situaciones educativas conflictivas tanto en el aula de clases como en el ámbito laboral en general y no logran encontrar las vías para la solución de esos problemas.

La segunda parte de la entrevista se dirigió a saber, de qué manera la institución educativa atiende el desarrollo de las competencias transversales en sus docentes:

- Refieren que la unidad educativa ha realizado acciones dirigidas al desarrollo de las competencias transversales de los docentes, fundamentalmente acciones de capacitación, y no solo dirigidas a las materias que ellos imparten sino a su comportamiento con los estudiantes. En todos los proyectos que tiene la escuela, se ha tenido en cuenta las debilidades y fortalezas de los docentes.

- Falta enseñarles a los colaboradores qué competencias transversales deben desarrollar, es decir, es necesario planificar y organizar acciones de capacitación pero dirigidas a apren- der, estudiar y profundizar en estas competencias transversales, pues nadie puede enseñar ni dar, lo que no posee.

- Las acciones se diseñan, se organizan, pero luego no se aplican y mucho menos se controlan.

- Piensan que ha faltado trabajo en equipo en las capacitaciones, estimular el trabajo entre todos y de uno con otros. La participación no siempre es alta ni se logra integración. Por ejemplo, la reunión de maestros de un mismo año es algo que no se ha logrado, para que dialoguen entre sí acerca de qué hay que hacer con los estudiantes, en conjunto.

Los resultados de la entrevista en profundidad realizada a las autoridades y a los informantes clave, confirman los resultados obtenidos con el resto de los instrumentos, lo que corrobora el estado alejado de lo deseado que presenta el desarrollo de las competencias transversales en los docentes de la unidad educativa, especialmente los que imparten clases al bachillerato unificado.

\section{Conclusiones}

El desarrollo de competencias transversales en los docentes del Bachillerato General Unificado en el Centro Educativo Miraflores tiene afectaciones, condicionalmente la mayoría de las frecuencias en que aparecen los valores a partir de los instrumentos aplicados, se encuentran entre las opciones de medio a bajo, estando las mayores insuficiencias en las competencias para la resolución de problemas, la iniciativa y la toma de decisiones.

Las competencias transversales de implicación responsable en el trabajo, la autonomía y el trabajo en equipo, tienen un alto desarrollo, lo que constituye la fortaleza que poseen estos docentes que facilitarían el desarrollo de las competencias afectadas, de manera de lograr una mejor influencia en la calidad de los aprendizajes de los estudiantes del Bachillerato General Unificado en el Centro Educativo Miraflores.

Con relación a las características particulares de las competencias, se presentaron insuficiencias en:

- El enfrentamiento y respuesta a las situaciones educativas que se le presentan a los docentes

- La organización de estrategias de solución, que facilitan actuar frente a dichas situaciones educativas 
- En la aplicación de estrategias de solución a las problemáticas a que se enfrenta.

- La disposición a la colaboración que presentan los docentes

- La ejecución exitosa de tareas en conjunto.

- El estímulo al trabajo en equipo, tan importante para lograr que los estudiantes se involucren activamente en el aprendizaje.

- La identificación de los problemas educativos, que permite encontrar donde estos se encuentran. :

- La identificación de las alternativas de solución viables que permitan dar solución a los problemas educativos.

- La implementación de las alternativas de solución.

- La evaluación de las alternativas de solución.

A partir de estos resultados, se llega a la conclusión de que la variable desarrollo de las competencias transversales de los docentes se encuentra alejada de lo deseado, con limitaciones en competencias para la resolución de problemas, la iniciativa, la autonomía y la toma de decisiones.

\section{Referencias bibliográficas}

1. Asamblea Constituyente. Constitución de la República del Ecuador [Internet]. Registro Oficial No. 449, 20 de octubre de 2008. Recuperado de https://goo.gl/9DHTXY

2. Presidencia de la República. Ley Orgánica de Educación Intercultural. Registro Oficial No. 417, 31 de marzo de 2011[Internet]. 2011. Recuperado de http://goo.gl/ZrDicQ

3. Ministerio de Educación. Estándares de Calidad Educativa. Aprendizaje, Gestión Escolar, Desempeño Profesional e Infraestructura. [Internet]. 2012. Recuperado de http://goo.gl/mEQEyF

4. Aguiar, M. Las competencias profesionales: algo más. Revista de Educación y Desarrollo [Internet]. 2005 Octubre Diciembre 4: 46-51. Recuperado de http://goo.gl/93pK6k

5. González, M., y Álvarez, Y. La formación de competencias profesionales del profesor: las competencias investigativas. Revista Electrónica de Desarrollo de Competencias (REDEC), [Internet](enero-junio de 2012). 5(1): 1-19. Recuperado de http://goo.gl/zwi7xd

6. Jofré, G. Competencias profesionales de los docentes de enseñanza media de Chile. Un análisis desde las percepciones de los implicados [Tesis doctoral]. 2009. (Universidad Autónoma de Barcelona, Barcelona, España). Recuperado de http://goo.gl/NqvYrY

7. Barriga, A. El enfoque de las competencias en la educación. ¿Una alternativa o un disfraz de cambio? Perfiles Educativos[Internet]. 2005. 28(111), 7-36. Recuperado de http://goo.gl/ZjUjzV

8. Solé-Pareta, J., Domingo-Pascual, J., y Santos-Boada, G. Actividades para fomentar las competencias transversales relacionadas con el razonamiento científico-técnico. Actas de las XX JENUI[Internet]. Julio 2014. Oviedo España. Recuperado de http://goo.gl/xOvCxH

9. Torrecilla, E., Martínez, F., Olmos, S., y Rodríguez, M. (mayo-agosto de 2014). Formación en competencias básicas para el futuro profesorado de educación secundaria: competencias informacionales de resolución de conflictos. Profesorado. Revista de Currículum y Formación de Profesorado[Internet]. Mayo-Agosto 2014. 18(2): 189-208. Recuperado de http://goo.gl/DEkY3q

10. Hinojosa, S. El desarrollo de competencias transversales en los docentes. Revista Electrónica Pedagogía Profesional,[Internet].Abril-Junio 2015. 13(2). Recuperado de http://www.pedagogiaprofesional.rimed.cu/Numeros/Vol13\%20no2/ susana.pdf 